University of Nebraska - Lincoln

DigitalCommons@University of Nebraska - Lincoln

5-15-2005

\title{
Spin glass or random anisotropy?: The origin of magnetically glassy behavior in nanostructured $\mathrm{GdAl}_{2}$
}

\author{
P. M. Shand \\ University of Northern lowa \\ C. C. Stark \\ University of Northern lowa \\ D. Williams \\ University of Nebraska-Lincoln \\ Marco Morales Torres \\ University of Nebraska - Lincoln, mmorales_torres2@unl.edu \\ T. M. Pekarek \\ University of North Florida, tpekarek@unf.edu
}

See next page for additional authors

Follow this and additional works at: https://digitalcommons.unl.edu/physicslesliepelecky

Part of the Physics Commons

Shand, P. M.; Stark, C. C.; Williams, D.; Morales Torres, Marco; Pekarek, T. M.; and Leslie-Pelecky, Diandra, "Spin glass or random anisotropy?: The origin of magnetically glassy behavior in nanostructured $\mathrm{GdAl}_{2}$ " (2005). Diandra Leslie-Pelecky Publications. 5.

https://digitalcommons.unl.edu/physicslesliepelecky/5

This Article is brought to you for free and open access by the Research Papers in Physics and Astronomy at DigitalCommons@University of Nebraska - Lincoln. It has been accepted for inclusion in Diandra Leslie-Pelecky Publications by an authorized administrator of DigitalCommons@University of Nebraska - Lincoln. 


\section{Authors}

P. M. Shand, C. C. Stark, D. Williams, Marco Morales Torres, T. M. Pekarek, and Diandra Leslie-Pelecky 


\title{
Spin glass or random anisotropy?: The origin of magnetically glassy behavior in nanostructured $\mathrm{GdAl}_{2}$
}

\author{
P. M. Shand and C. C. Stark \\ Department of Physics, University of Northern Iowa, 205 Physics Building, Cedar Falls, Iowa 50614 \\ D. Williams and M. A. Morales \\ Department of Physics and Astronomy and Center for Materials Research and Analysis, University \\ of Nebraska-Lincoln, Lincoln, Nebraska 68588-0111 \\ T. M. Pekarek \\ Department of Chemistry and Physics, University of North Florida, 4567 St. John's Bluff Road South, \\ Jacksonville, Florida 32224 \\ D. L. Leslie-Pelecky \\ Department of Physics and Astronomy and Center for Materials Research and Analysis, University \\ of Nebraska-Lincoln, Lincoln, Nebraska 68588-0111
}

(Presented on 11 November 2004; published online 12 May 2005)

Initially crystalline $\mathrm{GdAl}_{2}$ was mechanically milled for long times to produce a highly chemically disordered phase with approximately 8-nm grains. Analysis of dc magnetization measurements using an Arrott plot and the approach to saturation suggest the presence of significant random anisotropy. ac susceptibility measurements showed that the shift in the peak temperature with frequency usually seen in magnetically glassy and superparamagnetic systems was virtually undetectable in the $10-1000-\mathrm{Hz}$ frequency range. Based on these results, we believe that this material represents an interacting system with random anisotropy, where the anisotropy is the result of surface and interface asymmetries. (ㅇ 2005 American Institute of Physics.

[DOI: $10.1063 / 1.1853003$ ]

Bulk crystalline $\mathrm{GdAl}_{2}$ is a ferromagnet with a Curie temperature of $T_{C}=170 \mathrm{~K} .^{1}$ The ferromagnetism is due to the Ruderman-Kittel-Kasuya-Yosida (RKKY)-like exchange interactions among the localized $4 f \mathrm{Gd}$ atomic moments mediated by the itinerant $5 d$ and $6 s$ electrons. ${ }^{2,3}$ Amorphous $\mathrm{GdAl}_{2}$ apparently does not undergo a ferromagnetic (FM) transition; it remains paramagnetic (PM) down to $16 \mathrm{~K}$, where it becomes a spin glass (SG). ${ }^{4}$

Zhou and Bakker ${ }^{5}$ (ZB) have fabricated nanostructured $\mathrm{GdAl}_{2}$ by mechanical milling. The milling resulted in an average grain size of $20 \mathrm{~nm}$. In addition, it induced so-called quadruple-defect disorder in which $\mathrm{Al}$ atoms substitute for $\mathrm{Gd}$ in the $\mathrm{Gd}$ sublattice. Gd atoms do not substitute for $\mathrm{Al}$ atoms on the $\mathrm{Al}$ sublattice because of the ionic size difference. Hence, despite the significant positional disorder and vacancy formation, the system does not become amorphous even after extended periods of milling. In ZB's work, a FM transition still occurred for relatively short milling times, but a peak also developed in the magnetization at low temperatures. After $1000 \mathrm{~h}$ of milling, the FM phase had virtually disappeared and the low-temperature peak sharpened and shifted upward in temperature. ZB identified the new phase as a SG based upon ac susceptibility and dc magnetization measurements.

A low-temperature and low-field magnetic ground state with glassy characteristics is preferred in a vast number of disordered systems. ${ }^{6-13}$ It is difficult to experimentally establish the precise type of glassy ordering or blocking because many of the magnetic signatures are similar. There are, however, some subtle differences. The dynamics of the super- paramagnetic (SP) blocking process is different than that in spin glass-like (SGL) freezing. For example, the shift in the peak in the ac susceptibility with frequency is generally much larger in SP blocking processes than in SGL freezing.

In previous papers, ${ }^{14,15}$ the characteristics of the PM to FM transition in mechanically milled (mm-) $\mathrm{GdAl}_{2}$ were discussed. In this paper, dc magnetization and ac susceptibility are used to probe the nature of the low-temperature magnetic state in $\mathrm{mm}-\mathrm{GdAl}_{2}$. These results indicate that the lowtemperature magnetic behavior bears distinct similarities to that exhibited by random anisotropy magnets.

High-intensity milling of crystalline $\mathrm{GdAl}_{2}$ was performed in a SPEX 8000 mill with ball-to-powder mass ratio of 1:1.75. All sample handing was performed in an Ar atmosphere to prevent oxidation. An analysis of x-ray diffraction data using an integral breadth method showed a terminal mean grain size of $\sim 8 \mathrm{~nm}$, which was attained after $20 \mathrm{~h}$ of milling. Continued milling resulted in increasing disorder. A more extensive analysis of the structural changes can be found elsewhere. ${ }^{14}$ The dc magnetic measurements were made with a Quantum Design superconducting quantum interference device (SQUID) magnetometer and the ac susceptibility measurements with a Lakeshore susceptometer. Results were obtained for many different milling times, but the data in this paper will, unless otherwise noted, pertain to the sample milled for $400 \mathrm{~h}$.

Figure 1 shows an Arrott plot of the $M(H)$ data for $H$ $\leqslant 70 \mathrm{kOe}$ and $5 \mathrm{~K} \leqslant \mathrm{~T} \leqslant 130 \mathrm{~K}$. Note that there is strong curvature toward the $H / M$ axis beginning at moderate fields $(\sim 1 \mathrm{kOe})$, so there is no intercept on the $M^{2}$ axis. This sug- 


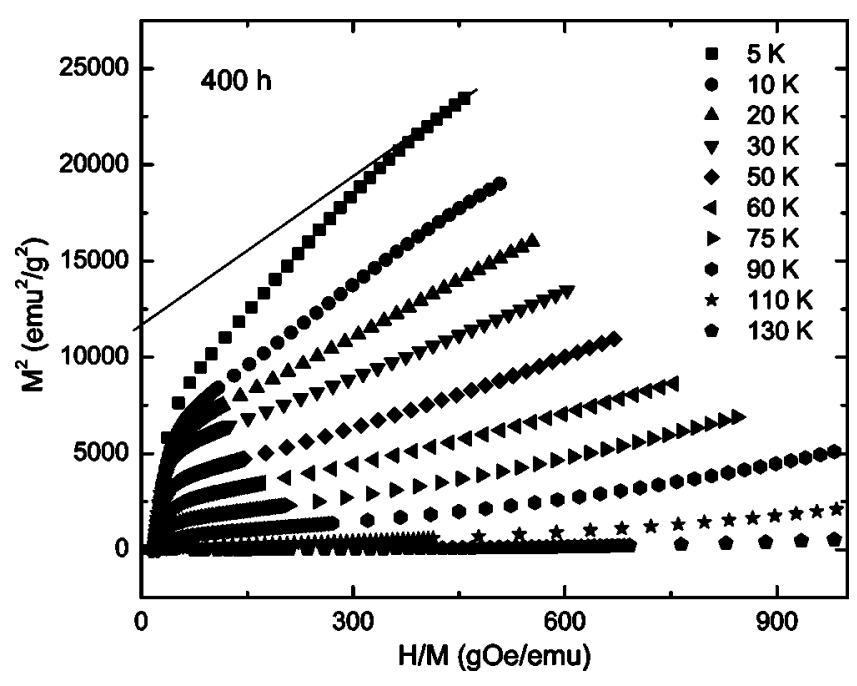

FIG. 1. Arrott plot using dc magnetization vs applied field $(H \leqslant 70 \mathrm{kOe})$ data taken at various temperatures for $\mathrm{GdAl}_{2}$ milled for $400 \mathrm{~h}$.

gests that the system exhibits no spontaneous magnetization. Extrapolating from high fields produces an intercept, which indicates a field-induced FM alignment. The intercept for the 5-K data (shown in Fig. 1) gives a corresponding spontaneous magnetization of $\sim \frac{3}{5}$ of the saturation magnetization. The strong curvature at low fields and the general shape of the Arrott plot is reminiscent of the behavior theoretically predicted by Aharony and Pytte (AP) of a random anisotropy system with weak anisotropy. ${ }^{16}$ AP predicted a transition from PM to an infinite susceptibility phase with no longrange magnetic order in zero field. If higher-order anisotropy terms are considered, the susceptibility is large but finite, i.e., the intercept on the $H / M$ axis is small, but nonzero. The data in Fig. 1 exhibit behavior very similar to the AP predictions. The AP theory has also been invoked for the interpretation of experimental results for the amorphous Gd-based alloys. ${ }^{17,18}$

Chudnovsky et al. developed a more extensive theory of ferromagnets with random anisotropy for a range of anisotropy and applied magnetic-field strengths. ${ }^{19}$ For the weakanisotropy case, there are three regimes, depending on the strength of the field $H$. The strength of $H$ is measured relative to a parameter $H_{s}$, where

$$
H_{s}=\frac{H_{r}^{4}}{H_{\mathrm{ex}}^{3}} .
$$

In Eq. (1), $H_{r}$ is the anisotropy field and $H_{\mathrm{ex}}$ is the exchange field. For low fields $H<H_{s}$, one has a correlated spin glass, which has a large susceptibility and is equivalent to the AP high-susceptibility phase. In the intermediate-field regime $H_{s}<H<H_{\text {ex }}$, the spins are nearly aligned. However, the random anisotropy causes the directions of the magnetization of locally correlated regions to vary. This regime is called the ferromagnet with wandering axis. In this regime, the magnetization approaches saturation as

$$
M=M_{0}\left[1-\frac{1}{15}\left(\frac{H_{S}}{H}\right)^{1 / 2}\right],
$$

where $M_{0}$ is the saturation magnetization. For high fields $H>H_{\mathrm{ex}}$, all spins are virtually aligned with the field and

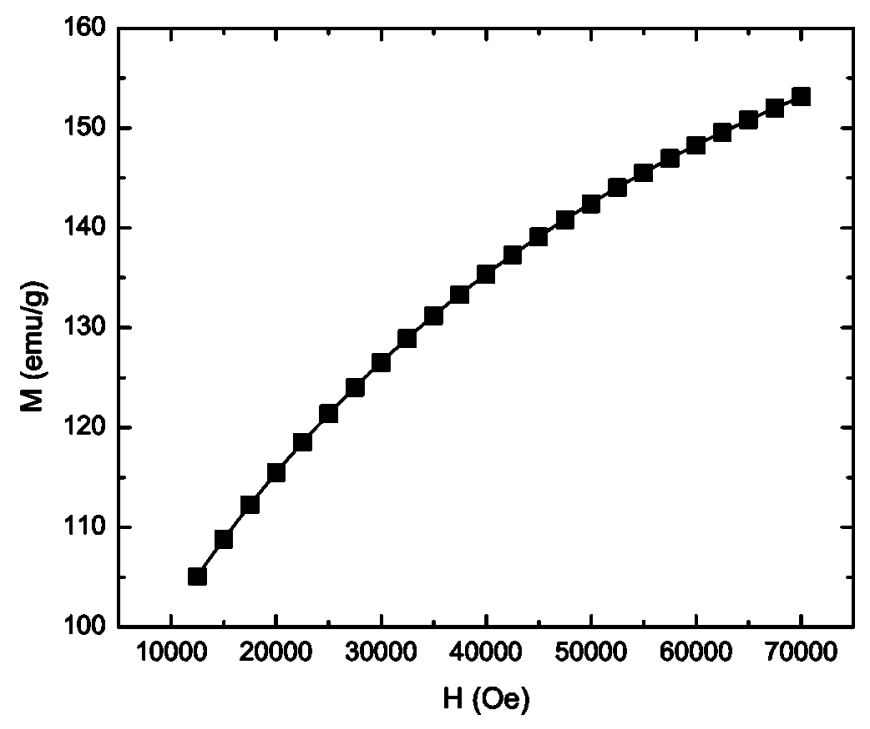

FIG. 2. de magnetization vs applied field data for $12 \leqslant H \leqslant 70 \mathrm{kOe}$ at $T$ $=5 \mathrm{~K} .70 \mathrm{kOe}$ is the maximum accessible field. The solid line is a fit to the theoretical expression for the approach to saturation in random anisotropy magnets at very large fields.

there is only a slight tipping angle due to the anisotropy. In this regime, $M$ is predicted to approach $M_{0}$ as

$$
M=M_{0}\left[1-\frac{1}{15}\left(\frac{H_{r}}{H+H_{\mathrm{ex}}}\right)^{2}\right] .
$$

In the preceding, we have ignored the effects of coherent anisotropy, which should be small in a Gd-based material.

In Fig. 2, a graph of $M$ vs $H$ for $12 \leqslant H \leqslant 70 \mathrm{kOe}$ is shown, along with a fit to Eq. (3). The fit is very good, with best-fit parameter values of $M_{0}=190 \mathrm{emu} / \mathrm{g}, H_{r}=292 \mathrm{kOe}$, and $H_{\mathrm{ex}}=100 \mathrm{kOe}$. The value of $M_{0}$ obtained from the fit is very close to the saturation magnetization value for pure $\mathrm{GdAl}_{2}$ (185 emu/g) using the spin-only value of the Gd moment. Surprisingly, $H_{r}>H_{\mathrm{ex}}$. However, as observed by Gehring et al., ${ }^{20}$ the prefactor $1 / 15$, which was calculated for $H_{r} \ll H_{\text {ex }}$, may not be precisely correct in general and may have to be modified when Eq. (3) is applied to real materials with somewhat higher levels of anisotropy. If the prefactor $\approx 1, H_{r}$ falls to $\sim 75 \mathrm{kOe}$. Note that the requirement $H$ $>H_{\text {ex }}$ for the validity of Eq. (3) was not met since our highest applied field was $70 \mathrm{kOe}$. However, the fit is good enough to suggest that for $\mathrm{mm}-\mathrm{GdAl}_{2}$, the applicability of Eq. (3) may extend to applied fields somewhat smaller than $H_{\text {ex }}$. The relatively high level of anisotropy that seems to characterize this system suggests that the low-temperature state where the anisotropy is likely strongest is speromagneticlike, i.e., the local magnetization is aligned along the local anisotropy axis. However, this state is not a microscopic speromagnet, since $H_{r}$ is probably less than or comparable to $H_{\mathrm{ex}}$. The more likely situation is that there are ferromagnetically correlated regions, the sizes of which are limited by the grain sizes, whose magnetization directions are pinned or frozen by random anisotropy. Perhaps the best description of the nature of this state is a cluster glass (CG). It should be mentioned that a fit to the data in Fig. 2 using Eq. (2) was quite poor. A much better fit was obtained over 


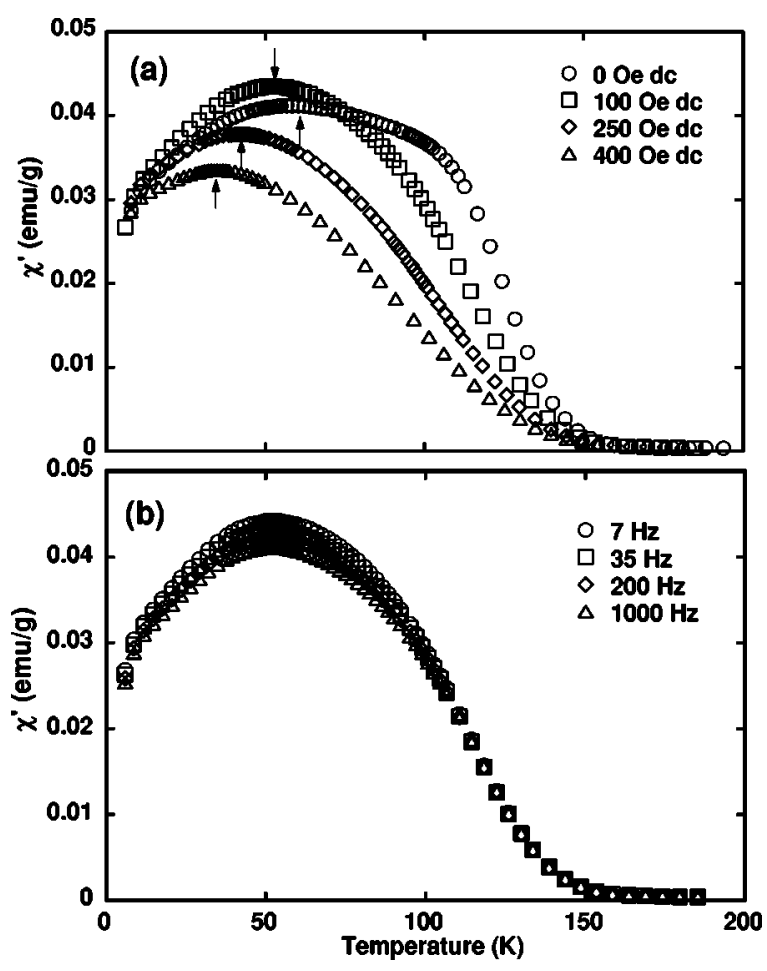

FIG. 3. ac susceptibility vs temperature for a sample milled for $590 \mathrm{~h}$. (a) The frequency is fixed at $200 \mathrm{~Hz}$, with various applied dc bias fields. (b) The dc bias field is fixed at $100 \mathrm{Oe}$, with various measuring frequencies.

the field range of $1 \leqslant H \leqslant 10 \mathrm{kOe}$. However, the extracted value of $M_{0}$ was $128 \mathrm{emu} / \mathrm{g}$, far short of the expected value of $185 \mathrm{emu} / \mathrm{g}$.

Shown in Fig. 3(a) are the data for the real part of the ac susceptibility $\left(\chi^{\prime}\right)$ as a function of temperature, taken at $200 \mathrm{~Hz}$ with several different values of the dc bias field. The sample was milled for $590 \mathrm{~h} . \chi^{\prime}$ shows strong suppression in the high-temperature region due to the alignment of FMcorrelated regions by the field. A peak is observed at low temperatures $(\sim 50 \mathrm{~K})$, which is consistent with CG-type ordering. $\chi^{\prime}$ falls more rapidly at the lowest temperatures. The peak is not sharp due to inevitable inhomogeneity in milled systems. The susceptibility at the peak is enhanced for bias fields up to $100 \mathrm{Oe}$ and then declines for stronger fields. The susceptibility enhancement for small bias fields is likely due to the reorientation of spins at the grain boundaries. ${ }^{21}$ The temperature at the peak decreases monotonically as the bias field increases. The peak temperature plotted as a function of $H^{-2 / 3}$ is linear, which is characteristic of the de AlmeidaThouless (AT) line in mean-field Ising spin glasses. ${ }^{9}$ This behavior has also been seen in interacting superparamagnets.

Figure 3(b) shows $\chi^{\prime}(T)$ for several different frequencies at a fixed dc bias field of 100 Oe. The magnitude of $\chi^{\prime}$ shows relatively weak dependence on the frequency and there is no discernible shift in the temperature at the peak with frequency. This rules out simple noninteracting SP grains and canonical SG behavior. If there is a relaxation time distribution (which is expected in a heterogeneous system such as
$\mathrm{mm}-\mathrm{GdAl}_{2}$ ), then only the tail of it lies inside the experimental time window, which extends over only somewhat greater than two decades. Very slow relaxation of the CG-like state could be due to strong frustration of FM alignment of the magnetization of the grains due to the disorder-induced random exchange and anisotropy within the grains and at the boundaries. Measurements at lower frequencies are necessary to verify this.

dc magnetization and ac susceptibility measurements have been performed on mechanically milled $\mathrm{GdAl}_{2}$. Arrottplot analysis and fitting $M(H)$ data with theoretical expressions describing the approach to saturation suggest a significant level of random anisotropy in mm-GdAl 2 ac susceptibility results are consistent with a cluster glasslike freezing of ferromagnetically ordered regions due to random anisotropy. We therefore conclude that as the temperature is lowered, $\mathrm{mm}-\mathrm{GdAl}_{2}$ first undergoes a transition from paramagnetism to a short-range-ordered FM state. At lower temperatures where the strength of the random anisotropy is higher, the magnetizations of the correlated regions freeze into a cluster glass state.

This work was supported by NSF Grant Nos. DMR9875425 and DMR-0305653, ACS Petroleum Research Fund Grant No. PRF\#40209-B5M, and the UNI College of Natural Sciences.

${ }^{1}$ A. Slebarski, Phys. Status Solidi 46, K29 (1978).

${ }^{2}$ E. Dormann, J. Magn. Magn. Mater. 6, 87 (1977).

${ }^{3}$ M. Bauer, M. S. S. Brooks, and E. Dormann, Phys. Rev. B 48, 1014 (1993).

${ }^{4}$ T. Mizoguchi, T. McGuire, R. Gambino, and S. Kirkpatrick, Phys. Rev. Lett. 38, 89 (1977).

${ }^{5}$ G. F. Zhou and H. Bakker, Phys. Rev. Lett. 73, 344 (1994).

${ }^{6}$ D. N. H. Nam, K. Jonason, P. Nordblad, N. V. Khiem, and N. X. Phuc, Phys. Rev. B 59, 4189 (1999); S. Mukherjee, R. Ranganathan, P. S. Anikumar, and P. A. Joy, Phys. Rev. B 54, 9267 (1996).

${ }^{7}$ K. H. Fischer and J. A. Hertz, Spin Glasses (Cambridge University Press, Cambridge, 1991).

${ }^{8}$ J. A. Mydosh, Spin Glasses: An Experimental Introduction (Taylor and Francis, London, 1993).

${ }^{9}$ K. Jonason, J. Mattsson, and P. Nordblad, Phys. Rev. B 53, 6507 (1996).

${ }^{10}$ V. Petkov, V. Skumryev, and A. Apostolov, Mater. Sci. Eng., A 133, 252 (1991).

${ }^{11}$ R. D. Zysler, C. A. Ramos, E. De Biasi, H. Romero, A. Ortega, and D. Fiorani, J. Magn. Magn. Mater. 221, 37 (2000).

${ }^{12}$ W. Kleeman, O. Petracic, Ch. Binek, G. N. Kakazei, Yu. G. Pogorelov, J. B. Souza, S. Cardoso, and P. P. Freitas, Phys. Rev. B 63, 134423 (2001).

${ }^{13}$ E. Bonetti, L. Del Bianco, D. Fiorani, D. Rinaldi, R. Caciuffo, and A. Hernando, Phys. Rev. Lett. 83, 2829 (1999).

${ }^{14}$ D. S. Williams, P. M. Shand, T. M. Pekarek, R. Skomski, V. Petkov, and D. L. Leslie-Pelecky, Phys. Rev. B 68, 214404 (2003).

${ }^{15}$ D. Williams, P. M. Shand, C. Stark, T. Pekarek, R. Brown, Lanping Yue, and D. L. Leslie-Pelecky, J. Appl. Phys. 93, 6525 (2003).

${ }^{16}$ A. Aharony and E. Pytte, Phys. Rev. Lett. 45, 1583 (1980).

${ }^{17}$ D. J. Sellmyer and S. Nafis, J. Appl. Phys. 57, 3584 (1985).

${ }^{18}$ B. Dieny and B. Barbara, J. Phys. (Paris) 46, 293 (1985).

${ }^{19}$ E. M. Chudnovsky, W. M. Saslow, and R. A. Serota, Phys. Rev. B 33, 251 (1986).

${ }^{20}$ P. M. Gehring, M. B. Salamon, A. del Moral, and J. I. Arnaudas, Phys. Rev. B 41, 9134 (1990).

${ }^{21}$ E. M. Levin, V. K. Pecharsky, and K. A. Gschneidner, J. Appl. Phys. 90, $6255(2001)$. 